\title{
Obstacle Height Estimation Related to Suitable Viewpoint while Waiting for the Bus Using Color Moment Technique
}

\author{
Watcharin Tangsuksant and Chikamune Wada
}

\begin{abstract}
To achieve the final goal of assisting blind people with obstacle detection along the roadside, the estimated height of obstacles is a crucial part of our proposed system, which is particularly important when waiting at bus stops. The color moment technique is used for matching the obstacle areas between two image areas. However, to distinguish between matching and non-matching images, it is necessary to establish certain criteria. This research proposes criteria selection and optimization based on different weight values used in a color moment equation and distance error measurement. Moreover, HSV and RGB color models were compared. The experimental results indicated that the optimal performance of the color moment technique with a weight of $(3,1,1)$ resulted in an accuracy up to $86 \%$ for the RGB color model. This high level of performance strongly suggests that our assistive application for blind people would be both feasible and beneficial.
\end{abstract}

Index Terms-Assistive application for blind, bus-waiting color moment, height of obstacle estimation, suitable viewpoints.

\section{INTRODUCTION}

Obstacle detection is one of the important research topics in the field of assistive applications. The primary objective in obstacle detection is to improve safety by alerting people to obstacles that may pose a threat. Generally, there are two main approaches to obstacle detection, including those that are sensor-based and others that are camera-based. Several types of sensor are usually used for obstacle detection, including ultrasonic, IR, and radar sensors, which are employed in smart walking sticks used by blind people to detect and avoid obstacles [1]-[4]. Further, many existing studies have applied an image processing technique to address obstacle detection and avoidance [4]-[6] using cameras. However, some research has combined both sensors and cameras to detect obstacles [7], [8], such as the Microsoft Kinect sensor [9].

For sensor-based applications, the smart walking stick is commonly used to assist blind people. For example, Nitish O. et al. [1] installed ultrasonic, IR, and other sensors with Arduino on a white stick for blind people to detect obstacles

Manuscript received May 16, 2019; revised February 1, 2020. This work was supported in part by the Graduate School of Life Science and Systems Engineering, Kyushu Institute of Technology and the Japanese Government (Monbukagakusho: MEXT) Scholarship.

Watcharin Tangsuksant and Chikamune Wada are with the Graduate School of Life Science and Systems Engineering, Kyushu Institute of Technology, Hibikino 2-4 Wakamatsu-ku Kitakyushu, Fukuoka 808-0196, Japan (e-mail: w.tangsuksant.m@hotmail.com, wada@brain.kyutech.ac.jp). in front of them. The audio message will alert blind users when the device detects the obstacles. Moreover, Mohammed A. T. [2] presented a smart walking stick that detects not only obstacles but also holes and water bodies. For such detection, an ultrasonic sensor was used to measure angles at about $40^{\circ}$. Moreover, a moisture sensor was installed on a leg of the stick to measure land soil moisture. Although the sensor-based configuration was deemed comfortable, it provides little information regarding obstacles that were in close proximity to the user.

Camera-based applications can detect and recognize the type of obstacles and provide more information than sensor-based designs. For example, Bor-Shing L. et al. [5] proposed object detection and recognition on a smartphone-based system for informing visually impaired people. They applied several machine learning techniques such as YOLO and Faster R-CNN. However, it could only detect seven types of object, such as bicycles, people, piers, and potted plants. Moreover, Johnson L. A. et al. [6] designed a wearable device. Their device converted visual information into tactile signals to guide visually impaired people and detect obstacles. In other studies, a depth camera, which combined the camera-based and IR sensors, was applied for obstacle detection. For example, Aladern A. et al. [8] proposed navigation assistance and obstacle detection using an RGB-D camera. The camera was hung on the user's neck while walking in a building. In addition, Paulo C. et al. [10] used a disparity image to detect potential object collision threats in indoor and outdoor environments. Their proposed method used a 2D ensemble empirical mode decomposition image optimization algorithm and two layers of depth image segmentation. However, it could only detect objects within $2 \mathrm{~m}$ because of the depth camera limitations.

Our application is different compared to existing research. The final goal is a smartphone application, which is portable and comfortable for blind people to get a suitable viewpoint while waiting for the bus at the roadside. In particular, this research focuses on an image matching technique to estimate the height of the obstacle along the road, which is a part of our system for obstacle detection.

\section{A. Background of Application and Suitable Viewpoints for Individuals Waiting for the Bus}

This proposed research involves an assistive application for blind individuals to detect obstacles from a suitable viewpoint when waiting for the bus. This section describes the background and definition of suitable viewpoints when waiting for the bus.

The suitable viewpoint definition of waiting for the bus was proposed in a previous research by W. Tangsuksant et al. [11], in which the camera was assumed to be held in the vertical axis. If the vanishing point of the perspective image 
falls in the range of $25 \%$ between $\mathrm{Vp}$ and the farthest left side in perfect camera panning, it will be defined as a suitable viewpoint, as shown in Fig. 1(a). Moreover, previous research proposed the classification of viewpoints for those waiting for the bus without considering obstacles. However, obstacles may appear in the images when the image is captured.

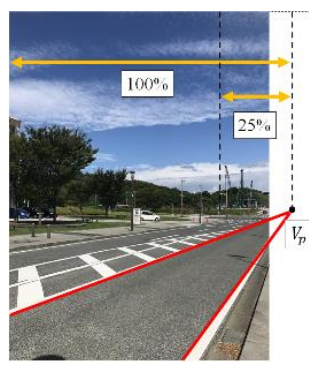

(a)

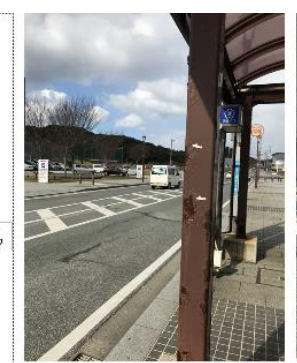

(b)

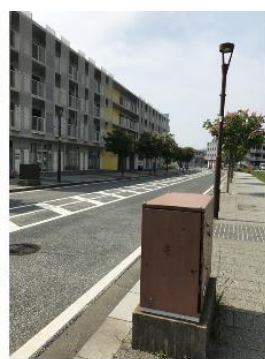

(c)
Fig. 1. (a) Suitable viewpoint definition, (b) unsuitable viewpoint with tall pole obstacles, and (c) suitable viewpoint with small obstacles

In real-world conditions, obstacles have various shapes and sizes. For example, the appearance of tall poles can obscure the suitable viewpoint for oncoming bus number detection, as shown in Fig. 1(b), whereas, the short obstacles in Fig. 1(c) do not obscure the suitable viewpoint for oncoming bus number detection. To achieve the final goal for our assistive application of suitable viewpoint classification, an obstacle consideration process is necessary.

There are two main processes of obstacle consideration along the road for our proposed system. The first step is to find the obstacle position along the road, and the second step is to estimate the height of the obstacles. For the first step, we proposed in our previous research [12] that a combined technique that includes image processing such as Hough Line Transformation, Uniform Local Binary Pattern, and vertical projection for finding the obstacle position is viable, as shown in Fig. 2.

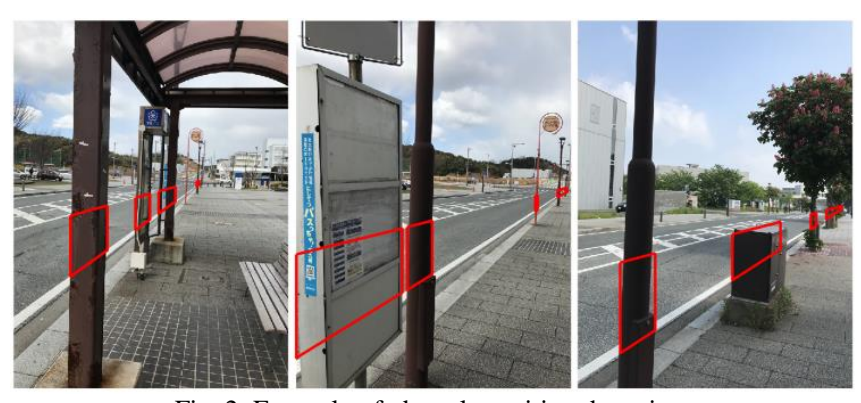

Fig. 2. Example of obstacle position detection.

For this research, estimating the height of obstacles has been considered. Although many obstacles can appear in an image, the proposed system considers only some of the obstacles that are in a range of interest of our system, which is related to the definition of a suitable viewpoint [11]. The system was designed with a range of interest (R-L) from $32.5 \%$ to $100 \%$ of the range, as shown in Fig. 3(a), which begins at $0 \%$, corresponding to the vanishing point of the perspective image. Then, estimation of obstacle height is performed by comparing areas 1 and 2, outlined with red squares, as shown in Fig. 3(b) and Fig. 3 (c). The system can detect area 1 in the first step of obstacle consideration. In addition, area 2 is an unknown area that is defined with the same square size as area 1 and located above the horizon line. The horizon line is a component of the perspective image [13], which is the line defined as eye level or camera position. Thus, we assume that the height of the obstacles has to be lower than the horizon line to maintain a suitable viewpoint for bus number detection.

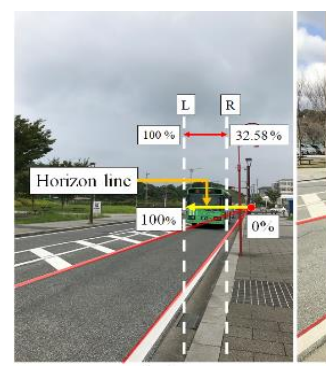

(a)

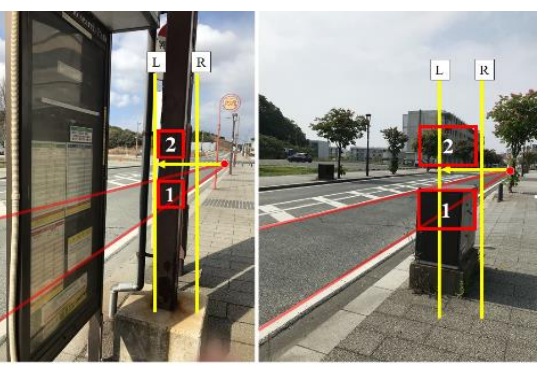

(b) (c)
Fig. 3. (a) Range of interest for obstacle consideration, (b) tall obstacle and range of interest, and (c) short obstacle and range of interest.

For our future work, we will apply this to the smartphone's application in real time, and thus, we intend to use a color moment technique that can simply and quickly estimate the height of obstacles along the road in daylight conditions. Moreover, this research emphasizes the optimization of the color model and weight selection. The remainder of this paper is organized as follows. Section II explains the concepts and proposed method of research. Section III reports the experiments and results of our proposed study. Moreover, the discussion and future work are presented in Section IV. Finally, the conclusion is presented in Section V.

\section{PROPOSED METHOD}

To estimate the height of the obstacles along the road, this research applies a color moment technique. Generally, the color moment technique [14] is used for image retrieval applications, which compares the unknown image and an image in a database using Eq. (1). The unknown image and the image in the database are represented by $H$ and $I$, respectively. Each $i$-th color channel is calculated by three moments that consist of mean $(E)$, standard deviation $(\sigma)$, and skewness $(s)$ as expressed in Eqs. (2), (3), and (4) with $j$-th image pixel $\left(p_{i j}\right)$. In addition, weight $(w)$ is a parameter that can be adjusted manually depending on the particular application.

$$
\begin{gathered}
d(H, I)=\sum_{i=1}^{r} w_{i 1}\left|E_{i}^{I}-E_{i}^{2}\right|+w_{i 2}\left|\sigma_{i}^{I}-\sigma_{i}^{2}\right|+w_{i 3}\left|s_{i}^{1}-s_{i}^{2}\right| \\
E_{i}=\frac{1}{N} \sum_{j=1}^{N} p_{i j} \\
\sigma_{i}=\left(\frac{1}{N} \sum_{j=1}^{N}\left(p_{i j}-E_{i}\right)^{2}\right)^{1 / 2} \\
s_{i}=\left(\frac{1}{N} \sum_{j=1}^{N}\left(p_{i j}-E_{i}\right)^{3}\right)^{1 / 3}
\end{gathered}
$$

Our application must distinguish the pair of images between matching and non-matching images, as shown in Fig. 3(b) and Fig. 3(c). Thus, this research proposes a simple mathematical model for clustering the category of the pair of images based on the color moment technique.

The concept consists of three main steps, as shown in Fig. 
4. First, the sample square images, which are two categories of matching and non-matching images from the real roadside scenarios, are collected by different sizes of the images depending on the step of obstacle position detection.

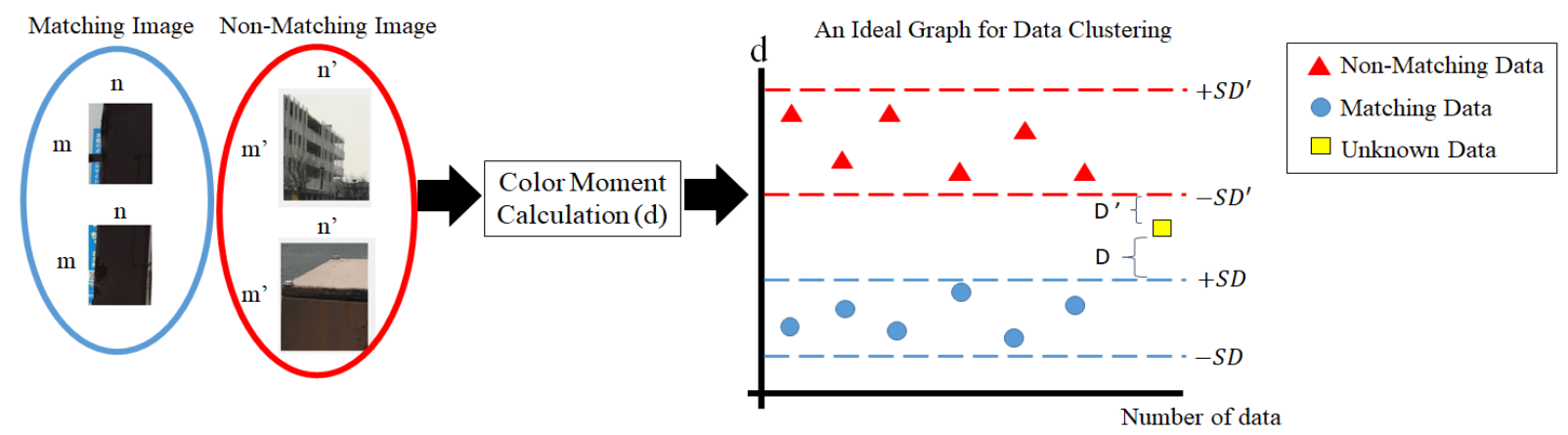

Fig. 4. The concept of the proposed method and an ideal graph for data clustering.

Then, the color moment in Eq. (1) is calculated for each pair of images for both categories. Finally, the standard deviation is calculated for both matching and non-matching data from the color moment outcome. For the ideal graph in Fig. 4, the calculated color moment of non-matching data will be higher than matching data because there are differences between pairs of images among non-matching data.

Moreover, this research proposes a simple method for clustering data between matching and non-matching images using the mean \pm standard deviation of each outcome data group. The $\pm S D^{\prime}$ and $S D$ lines separate the data between two categories, as shown in Fig. 4, where the $-S D^{\prime}$ value is higher than the $S D$ value. Then, when the unknown data are inducted into the system, system can use the criteria with Eq. (5) for data assignment. The outcome of the color moment (d) for non-matching data falls on the upper line of $-S D^{\prime}$, but the $\mathrm{d}$ of the matching data is lower than the $+S D$ line. Furthermore, in the case where $\mathrm{d}$ falls between the $-S D^{\prime}$ and $+S D$ lines, as shown in Fig. 4, it can be calculated by the distance $\left(D, D^{\prime}\right)$ between $\mathrm{d}$ and $-S D$ and $+S D$ as in Eq. (6).

$$
\begin{gathered}
\text { case }=\left\{\begin{array}{r}
\text { Non }- \text { Matching, } d \geq-S D^{\prime} \mid D^{\prime}<D \\
\text { Matching, } d \leq+S D
\end{array}\right. \\
D=\left|d-\left(-S D^{\prime}\right)\right|, D^{\prime}=|\mathrm{d}-(+S D)| .
\end{gathered}
$$

This section only proposes the feasibility of the concept for clustering the data using the color moment technique. However, it still has some points that we should consider, such as the type of the color model and the weight parameter of the color moment technique, as shown in Eq. (1). Therefore, this research will focus on this point for optimization.

\section{EXPERIMENTS AND RESULTS}

To optimize our proposed system, two experiments are considered in this section. First, HSV and RGB color models were selected because they are widely used for image processing. Second, the weight (w) of the color moment in Eq (1) has been optimized in many cases, and each case results in different outcomes.

Each channel of the HSV and RGB color models is calculated by three moments, including the mean (E), standard deviation $(\sigma)$, and skewness (s) for $\mathrm{H}$ and $\mathrm{I}$ of each image, which can be represented by matrices (7) and (8).

$$
\begin{gathered}
H_{h s v}=\left\lfloor\begin{array}{lll}
h_{E} & s_{E} & v_{E} \\
h_{\sigma} & s_{\sigma} & v_{\sigma} \\
v_{s} & s_{s} & v_{s}
\end{array}\right\rfloor, \quad I_{h s v}=\left\lfloor\begin{array}{ccc}
h_{E}^{\prime} & s_{E}^{\prime} & v_{E}^{\prime} \\
h_{\sigma}^{\prime} & s_{\sigma} & v_{\sigma}^{\prime} \\
v_{s}^{\prime} & s_{s}^{\prime} & v_{s}^{\prime}
\end{array}\right\rfloor \\
H_{r g b}=\left\lfloor\begin{array}{lll}
r_{E} & g_{E} & b_{E} \\
r_{\sigma} & g_{\sigma} & b_{\sigma} \\
r_{s} & g_{s} & b_{s}
\end{array}\right\rfloor, \quad I_{r g b}=\left\lfloor\begin{array}{lll}
r_{E}^{\prime} & g_{E}^{\prime} & b_{E}^{\prime} \\
r_{\sigma}^{\prime} & g_{\sigma}^{\prime} & b_{\sigma}^{\prime} \\
r_{s}^{\prime} & g_{s}^{\prime} & b_{s}^{\prime}
\end{array}\right\rfloor \\
w=\left\lfloor\begin{array}{lll}
w_{1} & w_{2} & w_{3} \\
w_{1} & w_{2} & w_{3} \\
w_{1} & w_{2} & w_{3}
\end{array}\right\rfloor
\end{gathered}
$$

For weight assignment, this research considers the effectiveness of each channel of the color model using the color moment technique, and thus, the weight $\left(w_{1}, w_{2}, w_{3}\right)$ can be assigned as matrix (9). To limit the integer number of weight values, 1, 2, and 3 were chosen for our experiment. Further, the weights were rearranged by permutations, excluding the cases of $(1,1,1),(2,2,2)$, and $(3,3,3)$. Therefore, there are 18 cases of weight testing for the optimization process.

In addition, this research provided two different datasets for calculating the criteria and employing the testing process. Each dataset contained 100 RGB and HSV images, where HSV was converted from an RGB color model [15], for matching and non-matching with different square sizes, as shown in Fig. 5(a) and Fig. 5(b), respectively, in daylight conditions.

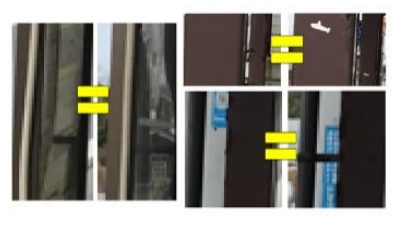

(a)

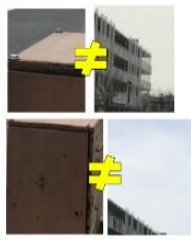

(b)

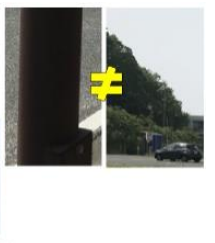

Fig. 5. (a) Example of matching images and (b) example of non-matching images.

\section{A. HSV and RGB Color Model Selection}

According to the color moment technique for image retrieval applications [13], an HSV color model was proposed. However, the RGB color model is also widely used for image processing. Therefore, this research compares both 
models in the experimental analysis.

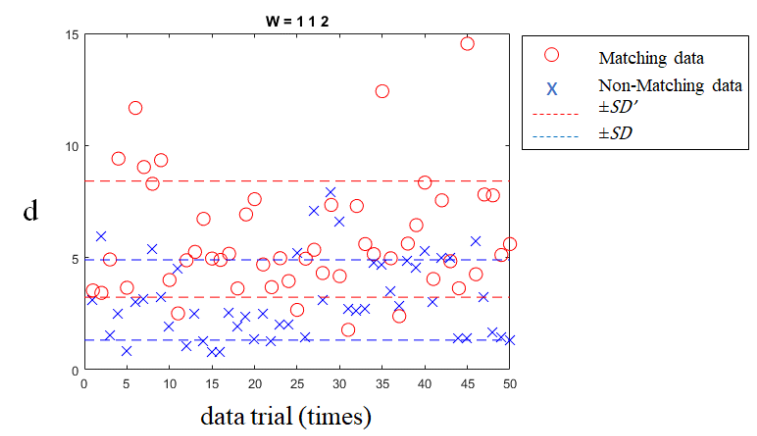

(a)

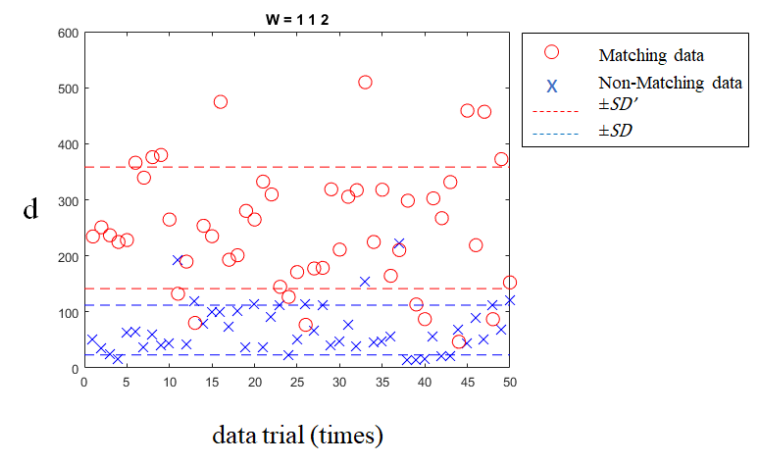

(b)

Fig. 6. (a) An example of an HSV color model with weight $(1,1,2)$ and (b) an example of an RGB color model with weight $(1,1,2)$.

Given the concept mentioned in Section II, the possible criteria must show $-S D^{\prime}>+S D$. For example, a comparison of the experimental results of the HSV and RGB color models with weight $(1,1,2)$ is presented in Fig. 6(a) and Fig. 6( (b), respectively. Obviously, the HSV model could not cluster the data between matching and non-matching images, whereas the RGB model performed rather well.

TABLE I: THE $-S D^{\prime}$ AND $+S D$ COMPARISON BETWEEN HSV AND RGB

\begin{tabular}{lllll}
\hline \hline & \multicolumn{3}{c}{ HSV } & \multicolumn{2}{c}{ RGB } \\
\cline { 2 - 5 } Weights & $-S D^{\prime}$ & $+S D$ & $-S D^{\prime}$ & $+S D$ \\
\hline $1,1,2$ & 3.23 & 4.90 & 107.78 & 83.57 \\
$1,2,1$ & 3.18 & 5.10 & 131.26 & 105.20 \\
$1,1,3$ & 3.94 & 6.00 & 111.64 & 85.22 \\
$1,3,1$ & 3.77 & 6.42 & 154.04 & 130.10 \\
$1,2,2$ & 4.08 & 6.15 & 131.26 & 105.20 \\
$1,3,3$ & 5.63 & 8.53 & 161.86 & 133.39 \\
$1,2,3$ & 4.86 & 7.24 & 139.04 & 108.47 \\
$1,3,2$ & 4.76 & 7.46 & 157.99 & 131.73 \\
$2,1,1$ & 3.01 & 5.53 & 172.44 & 141.03 \\
$2,2,1$ & 3.93 & 6.68 & 203.63 & 162.36 \\
$2,3,1$ & 4.67 & 7.91 & 232.31 & 185.04 \\
$2,1,3$ & 4.70 & 7.55 & 180.48 & 144.18 \\
$2,1,2$ & 4.79 & 7.62 & 176.49 & 142.60 \\
$3,1,1$ & 3.47 & 7.30 & 240.21 & 200.63 \\
$3,3,1$ & 5.36 & 9.54 & 303.41 & 242.76 \\
$3,1,2$ & 4.45 & 8.23 & 244.29 & 202.19 \\
$3,2,1$ & 4.50 & 8.38 & 272.63 & 221.18 \\
$3,1,3$ & 7.15 & 11.40 & 248.33 & 203.75 \\
\hline \hline
\end{tabular}

Table I shows the values of $-S D^{\prime}$ and $+S D$ for the HSV and RGB color models with different weights. Although the weights were changed, the $-S D^{\prime}$ values were still lower than the $+S D$ values in all cases, although $-S D^{\prime}>+S D$ was only shown by the RGB color model for all tested cases. Therefore, the RGB color model was selected for optimization in the next step.

TABLE II: PERformanCE OF RGB COLOR MOdEL TESTING BASED ON NON-TRANSPOSE MATRIX (H, I)

\begin{tabular}{llll}
\hline & \multicolumn{3}{c}{ Accuracy $(\%)$} \\
\cline { 2 - 4 } Weights & Matching images & Non-Matching Images & Average \\
\hline $1,1,2$ & 88 & 82 & 85 \\
$1,2,1$ & 86 & 82 & 84 \\
$1,1,3$ & 90 & 80 & 85 \\
$1,3,1$ & 86 & 80 & 83 \\
$1,2,2$ & 86 & 80 & 83 \\
$1,3,3$ & 90 & 80 & 85 \\
$1,2,3$ & 90 & 80 & 85 \\
$1,3,2$ & 86 & 80 & 83 \\
$2,1,1$ & 84 & 84 & 84 \\
$2,2,1$ & 84 & 84 & 84 \\
$2,3,1$ & 86 & 84 & 85 \\
$2,1,3$ & 86 & 82 & 84 \\
$2,1,2$ & 86 & 82 & 85 \\
$3,1,1$ & 84 & 84 & 84 \\
$3,3,1$ & 84 & 84 & 84 \\
$3,1,2$ & 84 & 84 & 84 \\
$3,2,1$ & 84 & 84 & 84 \\
$3,1,3$ & 86 & 82 & 85 \\
\hline \hline
\end{tabular}

\section{B. Weight Selection of Color Moment}

Although the RGB color model was chosen as shown in the previous experiment, a set of weights must be selected to optimize performance, and thus, this step is referred to as optimization for weight selection.

To optimize the performance of each weight value, a testing dataset of 100 pairs of images was used for matching and non-matching, which is different from previous datasets. Table II shows the performance of each weight testing, where the highest accuracy average is $85 \%$ for weights $(1,1,2),(1$, $1,3),(1,3,3),(1,2,3),(2,3,1),(2,1,2)$, and $(3,1,3)$.

TABLE III: PERFORMANCE OF RGB COLOR MODEL TESTING BASED ON

\begin{tabular}{llll}
\multicolumn{3}{c}{ TRANSPOSE MATRIX $\left(\mathrm{H}^{\prime}, \mathrm{I}^{\prime}\right)$} \\
\hline \hline & \multicolumn{3}{c}{ Accuracy $(\%)$} \\
Weights & Matching images & Non-Matching Images & Average \\
\hline $1,1,2$ & 86 & 82 & 84 \\
$1,2,1$ & 90 & 80 & 85 \\
$1,1,3$ & 86 & 82 & 84 \\
$1,3,1$ & 90 & 78 & 84 \\
$1,2,2$ & 90 & 80 & 85 \\
$1,3,3$ & 88 & 78 & 83 \\
$1,2,3$ & 90 & 80 & 85 \\
$1,3,2$ & 88 & 78 & 83 \\
$2,1,1$ & 88 & 80 & 84 \\
$2,2,1$ & 86 & 82 & 84 \\
$2,3,1$ & 88 & 80 & 84 \\
$2,1,3$ & 86 & 84 & 85 \\
$2,1,2$ & 88 & 82 & 85 \\
$\mathbf{3}, \mathbf{1}, \mathbf{1}$ & $\mathbf{8 8}$ & $\mathbf{8 4}$ & $\mathbf{8 6}$ \\
$3,3,1$ & 86 & 82 & 84 \\
$\mathbf{3 , 1 , 2}$ & $\mathbf{8 8}$ & $\mathbf{8 4}$ & $\mathbf{8 6}$ \\
$3,2,1$ & 86 & 82 & 84 \\
$3,1,3$ & 88 & 82 & 85 \\
\hline \hline
\end{tabular}

Although the experimental results displayed a relatively high accuracy, we attempted to further improve the accuracy of our proposed method. The $\mathrm{H}$ and I matrices of the RGB model described in (8) were modified by a transpose matrix $\left(H^{\prime}, I^{\prime}\right)$, as shown in (10). However, the same weights for testing were used in the previous experiment. Table III shows the improved performance by the highest accuracy (86\%) in case of $(3,1,1)$ and $(3,1,2)$ of weight testing. 


$$
\begin{gathered}
H_{r g b}^{\prime}=\left\lfloor\begin{array}{ccc}
r_{E} & r_{\sigma} & r_{s} \\
g_{E} & g_{\sigma} & g_{s} \\
b_{E} & b_{\sigma} & b_{s}
\end{array}\right], \quad I_{r g b}^{\prime}=\left[\begin{array}{ccc}
r_{E}^{\prime} & r_{\sigma}^{\prime} & r_{s}^{\prime} \\
g_{E}^{\prime} & g_{\sigma}^{\prime} & g_{s}^{\prime} \\
b_{E}^{\prime} & b_{\sigma}^{\prime} & b_{s}^{\prime}
\end{array}\right\rfloor \\
\bar{D}_{e r r}=\frac{1}{2}\left(\frac{1}{M}\left(\sum_{i=1}^{M} \mid\left(-S D^{\prime}\right)-E_{i}^{\prime}\right)+\frac{1}{N}\left(\sum_{i=1}^{N}\left|(+S D)-E_{i}\right|\right)\right) .
\end{gathered}
$$

TABLE IV: COMPARISON OF DISTANCE ERROR MEASUREMENTS BETWEEN

\begin{tabular}{cc} 
& WEIGHTS OF $(3,1,1)$ AND $(3,1,2)$ \\
\hline \hline weights & Distance error \\
\hline $3,1,1$ & 111.12 \\
$3,1,2$ & 112.32 \\
\hline \hline
\end{tabular}

Herein, weights $(3,1,1)$ and $(3,1,2)$ were considered particularly because of their highest degree of accuracy. Fig. 7 shows the plotted testing data and their criteria. In addition, some errors appeared in the plotted graph. For example, some matching data fell below the $+S D$ line, and some non-matching data fell above the $-S D^{\prime}$. To select the optimized weight for our application, the average distance error $\left(\overline{\mathrm{D}}_{\text {err }}\right)$ was measured for each error point (E), as shown in Eq. (11). $E_{i}^{\prime}$ and $E_{i}$ represent the error point for matching and non-matching data, respectively. Table IV shows the outcome of the distance error measurement between weights $(3,1,1)$ and $(3,1,2)$, where weight $(3,1,1)$ displayed an error value lower than weight $(3,1,2)$.

Therefore, weight $(3,1,1)$ of the transpose matrix for the RGB color model was selected for the implementation of the application in a future work.

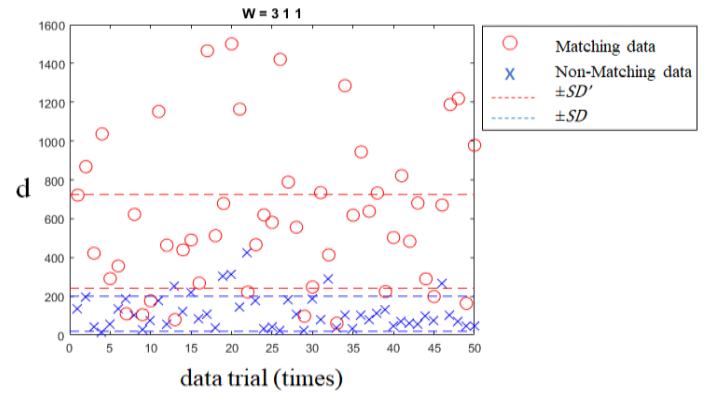

(a)

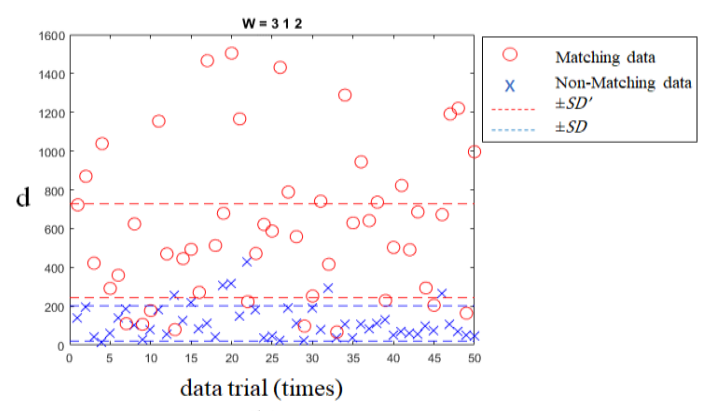

(b)

Fig. 7. (a) The transpose matrix testing with a weight of $(3,1,1)$ and (b) the transpose matrix testing with a weight of $(3,1,2)$.

\section{DISCUSSION AND FUTURE WORK}

\section{A. Discussion}

Two experiments consisting of color model selection and the weight of color moment selection were performed. In addition, this research also modified matrices $\mathrm{H}$ and $\mathrm{I}$ by the transpose matrices $H^{\prime}$ and $I^{\prime}$ to improve the accuracy of the proposed method.

On the basis of the experimental results, the RGB color model was able to distinguish $\left(-S D^{\prime}>+S D\right)$ between the data group of the matching and non-matching images in all cases of weight testing, but the results of HSV color model testing were totally dissimilar compared to the RGB modeling results.

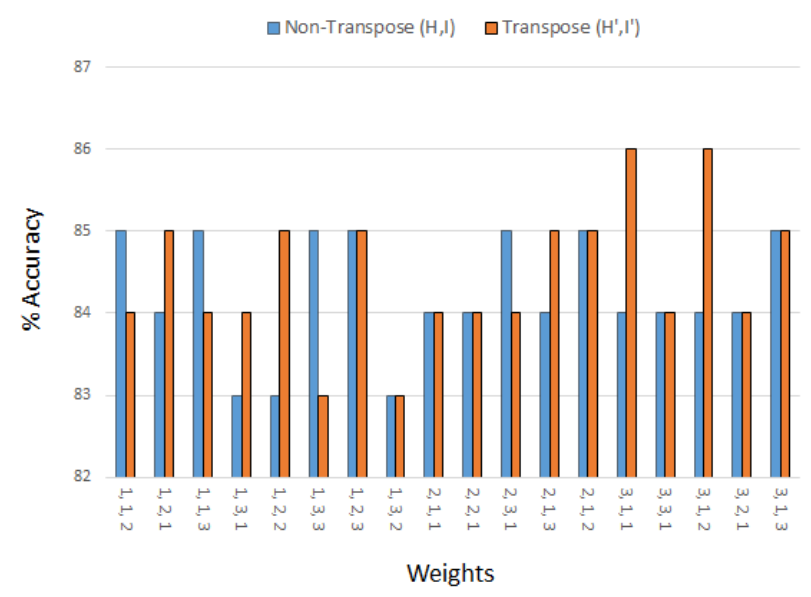

Fig. 8. Accuracy comparison between non-transpose (H, I) and transpose (H', I') matrices.

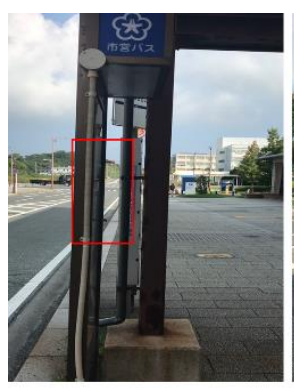

(a)

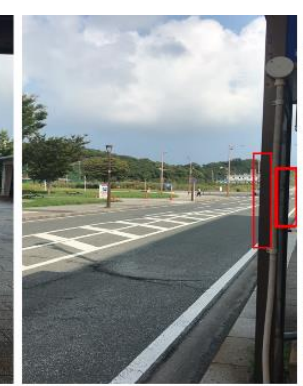

(b)

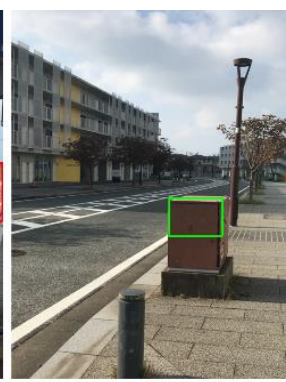

(c)
Fig. 9. (a) and (b) The detection of height obstacles with red boxes. (c) The detection of a short obstacle with a green box.

Furthermore, 18 cases of weight were tested using the RGB color model. First, matrices $\mathrm{H}$ and $\mathrm{I}$ in (8) were calculated, and the results showed the highest accuracy of $85 \%$ for many cases of weight testing. Second, the transpose matrices $H^{\prime}$ and $I^{\prime}$ in (10) were used to further improve the accuracy, and the highest accuracy was $1 \%$ higher than the results of the $\mathrm{H}$ and $\mathrm{I}$ matrix testing. Fig. 8 compares the accuracies of the non-transpose and transpose matrices, which resulted in the improvement in many cases, as shown for weights $(1,2,1),(1,3,1),(1,2,2),(2,1,3),(3,1,1)$, and $(3,1,2)$. Particularly in weight cases of $(3,1,1)$ and $(3,1,2)$, which showed the highest accuracy of $86 \%$, use of the transpose matrices resulted in performance that was better than all cases employing non-transpose matrices. As a result, the mean (E) moment for each channel of RGB had a significant effect on performance. Although the standard deviation $(\sigma)$ and skewness (s) were less significant than the mean, the distance error increased when the skewness was defined by 2 , as shown in Table IV. Fig. 9 shows the detection of obstacles along the road by our proposed method in real-world daytime conditions. The red boxes, shown in Fig. 9(a) and Fig. 9(b), identify the obstacle height and deem the location as unsuitable to wait for the bus, and the green 
box in Fig. 9(c) identifies a short obstacle along the road that does not obscure the suitable viewpoint and location to wait for the bus. However, this proposed method is still limited with respect to its application in nightlight conditions because of extreme darkness and differences in illumination.

\section{B. Future Work}

Although the experimental results showed good performance with a high degree of accuracy, the obstacle detection process was also necessary to apply for conditions at night, and in a future work, we will consider obstacle detection in nighttime conditions. We will try to adjust the suitable weight of color moment or find other complex techniques that can be applied well for nighttime conditions because it is quite difficult to detect obstacles in uncontrolled environments such as dark outdoor scenarios.

\section{CONCLUSION}

This research proposes the optimization of a color moment technique that will be applied to estimate obstacle height related to suitable viewpoints and locations to wait for the bus along the roadside in daylight conditions. First, HSV and RGB color models with 18 different weights were tested for our proposed method. The RGB color model provided feasible results, whereby $-S D^{\prime}>+S D$ was shown by all weights tested. Second, all cases of weight testing for the RGB model were optimized by accuracy measurements. In addition, this research compares the non-transpose and transpose matrices of $\mathrm{H}$ and I for the proposed color moment technique, and the results indicated that the transpose matrix performed the best with an accuracy of $86 \%$.

Finally, the RGB color model with a weight of $(3,1,1)$ for the transpose matrix was selected for application in our future research. However, it still has the limitation of not being usable in nighttime conditions, which we are planning to consider in our future work.

\section{CONFLICT OF INTEREST}

The authors declare no conflict of interest.

\section{AUTHOR CONTRIBUTIONS}

All authors have contributed in implementing, experimental design, analyzing the data. All authors have read and approved the final manuscript.

\section{REFERENCES}

[1] N. Ojha, P. K. Pradhan, and M. V. Patil, "Obstacle sensing walking stick for visually impaired," International Research Journal of Engineering and Technology (IRJET), vol. 4, no. 4, pp. 940-942.

[2] M. A. Therib, "Smart blinding stick with holes, obstacles and ponds detector based on microcontroller," Journal of University of Babylon, vol. 25, no. 5, pp. 1759-1768, 2017.

[3] A. Anwar and S. Aljahdali, "A smart stick for assisting blind people," IOSR Journal of Computer Engineering, vol. 19, no. 3, pp. 86-90, 2017.

[4] H. Sharma, M. Tripathi, A. Kumar, and M. S. Gaur. "Embedded assistive stick for visually impaired persons," in Proc. 9th International Conference on Computing, Communication and Networking Technologies (ICCCNT), IEEE, 2018, pp. 1-6.
[5] B. S. Lin, C. C. Lee, and P. Y. Chiang, "Simple smartphone-based guiding system for visually impaired people," Sensors, vol. 17, no. 6, p. $1371,2017$.

[6] L. A. Johnson and C. M. Higgins, "A navigation aid for the blind using tactile-visual sensory substitution," in Proc. 28th Annual International Conference of the IEEE Engineering in Medicine and Biology Society EMBS'06., 2006, pp. 6289-6292.

[7] N. Mohajeri, R. Raste, and S. Daneshvar, "An obstacle detection system for blind People," in Proc. the World Congress on Engineering, 2011, p. 2.

[8] A. Aladren, G. López-Nicolás, L. Puig, and J. J. Guerrero, "Navigation assistance for the visually impaired using RGB-D sensor with range expansion," IEEE Systems Journal, vol. 10, no. 3, pp. 922-932, 2016.

[9] H. H. Pham, T. L. Le, and N. Vuillerme, "Real-time obstacle detection system in indoor environment for the visually impaired using microsoft kinect sensor," Journal of Sensors, 2016.

[10] P. Costa, H. Fernandes, P. Martins, J. Barroso, and L. J. Hadjileontiadis, "Obstacle detection using stereo imaging to assist the navigation of visually impaired people," Procedia Computer Science, vol. 14, pp. 83-93, 2012

[11] W. Tangsuksant and C. Wada, "Classification of viewpoints related to bus-waiting for the assistance of blind people," International Journal of New Technology and Research (IJNTR), vol. 4, no. 9, pp. 43-52.

[12] W. Tangsuksant and C. Wada, "Static obstacle detection along the road with a combined method," in Proc. IEEE International Proceedings of the Biomedical Engineering International Conference, 2018.

[13] University of Utah and Department of Mathematics. (2007). The Geometry of Perspective Drawing on the Computer. [Online] Available: https://www.math.utah.edu/ treiberg/Perspect/Perspect.htm

[14] M. A. Stricker and M. Orengo, "Similarity of color images," Storage and Retrieval for Image and Video Databases III, vol. 2420, pp. 381-393, International Society for Optics and Photonics, 1995.

[15] A. R. Smith, "Color gamut transform Pairs," International Proceedings of ACM Siggraph Computer Graphics, vol. 12, no. 3, pp. 12-19, 1978

Copyright $\odot 2020$ by the authors. This is an open access article distributed under the Creative Commons Attribution License which permits unrestricted use, distribution, and reproduction in any medium, provided the original work is properly cited (CC BY 4.0).

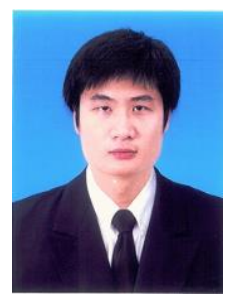

Watcharin Tangsuksant was born in Chumphon, Thailand, in 1990. He received his B.Eng. degree in biomedical engineering from Srinakharinwirot University, Bangkok, Thailand in 2013 and his M. Eng. degree in biomedical engineering from King Mongkut's Institute of Technology Ladkrabang, Bangkok, Thailand in 2015.

He was a lecturer at Rangsit University, Pathum Thani, Thailand in 2016, and he is currently a Ph.D. student of the Graduate School of Life Science and System Engineering, Kyushu Institute of Technology, Japan. His research interests include image processing, signal processing, and assistive technology for disabled people.

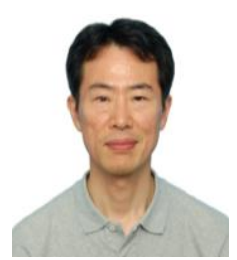

Chikamune Wada was born in Itami, Hyogo, Japan in 1966. He received his B.Eng. degree in mechanical engineering from Osaka University, Japan, in 1990 and his Ph.D. degree in biomedical engineering from Hokkaido University, Japan, in 1996.

From 1996 to 2001, he was an assistant professor with the Sensory Information Laboratory, at Hokkaido University. In 2001, he became an associate professor with Human-Function Substitution System Laboratory, Kyushu Institute of Technology. Since 2016, he has been a professor with the Human-function Substitution System Laboratory. His research interests include assistive technology, especially measuring human motion and informing disabled people of the necessary information to improve their QOL.

Prof. Chikamune Wada is one of the chief editors for the journals of the Institute of Electronics, Information and Communication Engineers (IEICE). 